\title{
A Network Technique Based Feature Extraction Method For Remote Sensing Images
}

\author{
Wei Xia \\ China Transport Telecommunications \& Information Center \\ Beijing 100011, China
}

\begin{abstract}
For hyperspectral remote sensing, the dataset usually contains hundreds of spectral images, which generates a rather large amount of data. To reduce the computational complexity of image analysis, feature extraction is often adapted. This paper presents a new approach for unsupervised feature extraction by transforming the hyperspectral dataset into complex networks. The networks' statistical topological properties are investigated to evaluate remote sensing image features. The objective of the method is to find the images which can form the most representative network formation. This is a completely new criterion for feature selection. Meanwhile, the proposed technique has both an explicit physical meaning. Experimental results demonstrate that the method achieves better results with respect to traditional methods.
\end{abstract}

Keywords-remote sensing; complex network analysis; feature extraction; topology characteristic

\section{INTRODUCTION}

The hyperspectral remote sensing produces images by hundreds spectral bands, with nano-scale spectrum resolution [1]. If all the spectra bands are concerned, there will be huge information for computer to calculate. Therefore, feature extraction (also named band selection), a process to choose the relevant range of wavelengths in the spectra while avoid losing accuracy for given task, is frequently adopted as a preprocessing step in hyperspectral imagery processing. An accurate feature extraction results encourages efficient results for hyperspectral analysis, such as target detection, classification, and so on.

Traditional feature extraction methods mainly focus on finding the most characteristic bands. During the last few years, researchers investigated different measures to discriminate the bands. The spatial distance, correlation coefficient, variance, and mutual information have been widely used as the criteria for lots of methods. For example, information divergence (ID) [2] uses the divergence criterion to quantify the difference between the probability distribution of a band image and its corresponding Gaussian probability distribution, selecting the spectral bands which contribute the largest divergences. The criteria of variance and correlation coefficient are used in maximum-variance principal component analysis (MVPCA) [3]. This method investigates the variances associated to the eigenvalues and eigenvectors of the hyperspectral bands, choosing the set of images with larger variances and lower correlation coefficient. The geometric algorithm for feature selection (GFS) [4] method, using geometric spatial distance to distinguish the bands, utilize geometry spatial mathematical method to obtained promising results. The above methods can select characteristic bands from the hyperspectral dataset, but it is not sure whether the results can maintain the intrinsic structure comparing to the original data.

This paper deals with the task of feature extraction in a different measurement, the network topology. By representing a series of signals through complex web, researchers are able to explore the inherent features form network organization. Such statistical features can provide new information about the phase space geometry, and enable the researchers to quantify and distinguish different dynamical regime [5]. In order to realize the topological analysis on hyperspectral remote sensing signals, the data are converted to the form of complex networks, and then the network topology is investigated to analyze the essential structure of the network. We construct networks both for the spectral band image and for the original data, selecting the bands which can form the most approximate network formation comparing with the original data.

\section{THE CONCEPTS OF NETWORKS}

This section introduces the basic definitions and notations for the complex network topology and the notations involved.

A complex network can be represented as a graph (or a web), presented as $\mathrm{G}=(\mathrm{V}, \mathrm{E})[6]$. A graph $\mathrm{G}=(\mathrm{V}, \mathrm{E})$ includes two sets $\mathrm{V}$ and $\mathrm{E}$. Here, the set $V=\left\{v_{1}, v_{2}, \ldots, v_{N}\right\}$ are the vertices (or nodes) of the graph, and $E=\left\{e_{1}, e_{2}, \ldots, e_{N}\right\}$ denote the edges (or links). In the graph, A Node $v_{i}$ can be referred by its order $i$ in the set $V$. Each of the edges is defined by a couple of nodes $i$ and $j$, and the link is represented by the notation $(i, j)$ or $l_{i j}$. The number of elements in $V$ and $E$ are noted as $\mathrm{N}$, and $\mathrm{K}$, respectively.

Usually, graph $\mathrm{G}=(V, E)$ can be described by a $\mathrm{N} \times \mathrm{N}$ size adjacency matrix $\mathbf{A}$. The entry of matrix $\mathbf{A}$ satisfies the following equation:

$$
a_{i j}=\left\{\begin{array}{ll}
1, & \text { if the link } e_{i j} \text { exists } \\
0, & \text { otherwise }
\end{array} .\right.
$$

For undirected graphs, $\mathbf{A}$ is a symmetric matrix. 
The number of edges of a node $i$ is defined as the degree $k_{i}$, satisfying the following equation:

$$
k_{i}=\sum_{j \in V} a_{i j}
$$

\section{THE PROPOSED METHOD}

In this section, the proposed method is introduced.

The developed complex network structures can describe a rather wide kind of different systems, and can enrich the knowledge gathered from both linear and nonlinear approaches, which can enable researchers to quantify and distinguish different dynamical regime [7]. However, in order to explore the inherent features of the data form network organization, one must represent the hyperspectral data through the complex webs.

\section{A. Build the Complex Network}

By treating the hyperspectral data as a series of signals, one can establish a bridge between the data between complex networks. Given a set of discrete signals $\left\{x_{i}\right\}_{i=1,2, \ldots N}$ of $\mathrm{N}$ observations, Two nodes $i$ and $j$ are connected if the following criterion is fulfilled:

$$
\begin{aligned}
& x_{i}, x_{j}>x_{n} \\
& \text { for all } n \text { such that } i<n<j
\end{aligned}
$$

This is called the horizontal visibility graph (HVG) [8], which is a simplification of the visibility graph algorithm [9]. It is worth noting that there are many other algorithms to transform series signals to networks, but the HVG has a feature that it is invariant under affine transformations of the series data. In other words, the rescaling of both horizontal and vertical axes will not affect the visibility criterion. This could be beneficial for investigating the inherent construction of hyperspectral image signals, so this paper chooses HVG as the observation network.

\section{B. Select the Feature Images/Bands}

In order to select the desired spectral bands, one need to search for the most "representative" bands. The network structure is quantified via a number of topological statistics, so one can distinguish different hyperspectral band by exploring the inherent features form network organization.

Actually, there are many different statistics that can be used to provide the information for distinguishing different networks. For the sake of simplicity, this paper just use one topological characteristic, the clustering coefficient [6], which is defined by:

$$
c_{i}=\frac{\sum_{j, m} a_{i j} a_{i m} a_{m i}}{k_{i}\left(k_{i}-1\right)}
$$

Here, $c_{i}$ is the clustering coefficient of the node $\mathrm{i}$, while $\mathrm{j}$ and $m$ are the neighbors of node $i$.

The coefficient $c_{i}$ represents the clustering properties of a web, so one can use it to quantify the organization of the complex network generated from different band subsets.

\section{The Procedure of the Proposed Algorithm}

Based on the above discussion, the detailed procedures of for feature extraction approach is summarized as follows:

Algorithm: Network-technique-based Band Selection (NBS)

Given the observation hyperspectral images and the number of bands to be selected.

1) For each spectral band,

a) Reshape the hyperspectral images to be series data.

b) Build the complex network by using visibility connecting criterion (3)

c) Calculate clustering coefficient $c_{i}$ by using (4), and compare $c_{i}$ with that of all the other spectral bands.

2) Choose the bands whose average clustering coefficient $C_{i}$ is more distinctive than others'. Those bands have more distinguishing network organization.

3) Reduce the dimension of original da according to the selected bands

\section{THE EXPERIMENTAL RESULTS}

In order to verify the effectiveness of the proposed algorithm, this section compares NBS algorithm with other three typical algorithms, including ID [2], MVPCA [3], and GFS [4]. The test dataset is the Indian pines AVIRIS data, which has been widely used for remote sensing experiments. The dataset was captured from Northwestern Indiana by Airborne Visible/Infrared Imaging Spectrometer (AVIRIS) in June 1992 [10][11]. This dataset consists a total of 224 bands with the spectral resolution of $10 \mathrm{~mm}$, covering the wavelength range of $0.4 \sim 2.5 \mu \mathrm{m}$. The spatial resolution of the Indian dataset is $17 \mathrm{~m}$.

Before selecting the spectral bands, the noisy or waterabsorption bands (including band 1 4, band 103 113, and band 148 166) are removed from the original dataset, leaving a number of 186 spectral bands. In order to evaluate the performance of these methods, two classifiers, K-nearest neighbourhood (KNN) [12], support vector machine (SVM) [13] are used for testing the impacts of feature extraction on the classification. Here, the ratio between training samples with testing samples is set as 1:5. 
The numbers of selected band ranges are changed from 8 to 14, and the corresponding classification results of SVM and KNN are shown in Figure I and Figure II, respectively. It can be found that when the number of selected bands is increasing, the classification accuracy of all the methods is enhancing. Generally speaking, the results on testing data obtained by the proposed method are better than that of GFS, ID and MVPCA. The results indicate that NBS method is a promising method for feature selection of hyperspectral remote sensing imagery.

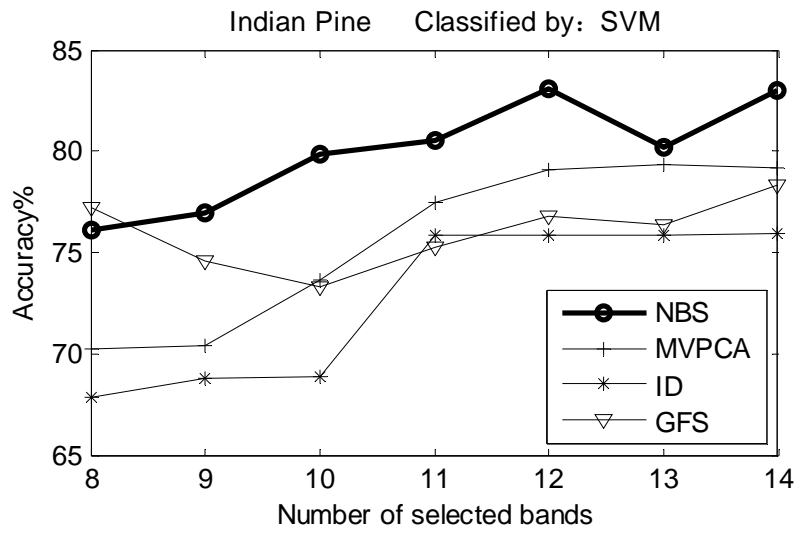

FIGURE I. CLASSIFICATION ACCURACIES USING THE SVM CLASSIFIER WITH DIFFERENT SELECTED BANDS.

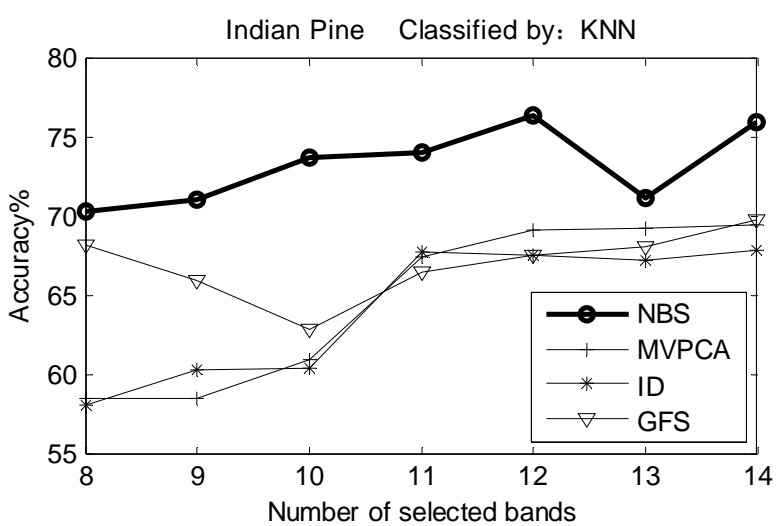

FIGURE II. CLASSIFICATION ACCURACIES USING THE KNN CLASSIFIER WITH DIFFERENT SELECTED BANDS.

\section{CONCLUSION}

This paper presents a new approach for unsupervised feature extraction. The proposed NBS method selects hyperspectral feature images by transforming the observation signals into the complex network and analyzing the corresponding topological characteristics. The net statistical properties are investigated to evaluate different bands. This is a novel criterion for band selection.

The NBS approach has several advantages. This method can maintain the topology features and intrinsic structure comparing to the original hyperspectral images. The method has both explicit physical meaning and a simple process. Experimental results show that the proposed approach can acquire better results with respect to traditional algorithms.

\section{REFERENCES}

[1] C.-I. Chang, Hyperspectral Imaging: Techniques for Spectral Detection and Classification, New York: Plenum, 2003.

[2] C.-I. Chang and S. Wang, "Constrained band selection for hyperspectral imagery," IEEE Trans. Geosci. Remote Sens., vol. 44, no. 6, pp. 15751585, Jun. 2006

[3] C.-I. Chang, Q. Du, T.-L. Sun, and M. Althouse, "A joint band prioritization and band-decorrelation approach to band selection for hyperspectral image classification," IEEE Trans. Geosci. Remote Sens., vol. 37, no. 6, pp. 2631-2641, Nov. 1999.

[4] L. Wang, X. Jia, and Y. Zhang, "A novel geometry-based feature selection technique for hyperspectral imagery," IEEE Geosci. Remote Sens. Lett., vol. 4, no. 1, pp. 171-175, 2007.

[5] J. Zhang, J.-F. Sun, X.-D. Luo, K. Zhang, T. Nakamura, M. Small, "Characterizing pseudoperiodic time series through the complex network approach," Physica D, vol. 23, pp. 2856-2865, 2008.

[6] S. Boccaletti , V. Latora , Y. Moreno , M. Chavez and D. U. Hwang, "Complex networks: Structure and dynamics," Phys. Rep., vol. 424, p. $175,2006$.

[7] J. Zhang, M. Small, "Complex network from pseudoperiodic time series: Topology versus dynamics,” Phys. Rev. Lett., vol. 96, pp. 238701, 2006.

[8] B. Luque, L. Lacasa, F. Ballesteros, J. Luque, "Horizontal visibility graphs: exact results for random time series," Phys. Rev. E, 80, p. 046103, 2009.

[9] L. Lacasa, B. Luque, F. Ballesteros, J. Luque, J. C. Nuno, "From time series to complex networks: the visibility graph," Proc. Natl. Acad. Sci., vol. 105, no.13, pp. 4972-4975, 2008.

[10] Available at http://speclab.cr.usgs.gov/spectral.lib04/lib04-AVIRIS.html

[11] D. Landgrebe, Multispectral data analysis: A signal theory perspective. West Lafayette: School of Electrical \& Computer Engineering, Purdue University, pp.56-89, 1998.

[12] T. Cover and P. Hart, "Nearest neighbor pattern classification," IEEE Trans. Inf. Theory, vol. 13, no, 1, pp. 21-27, Jan. 1967.

[13] F. Melgani and L. Bruzzone, "Classification of hyperspectral remote sensing images with support vector machines," IEEE Trans. Geosci. Remote Sens., vol. 42, no. 8, pp. 1778-1790, Aug. 2004. 